Published in final edited form as:

Exp Cell Res. 2008 May 1; 314(8): 1860-1868.

\title{
Suppression of sphingomyelin synthase 1 by small interference RNA is associated with enhanced ceramide production and apoptosis after photodamage
}

\author{
Duska Separovic ${ }^{a, b},{ }^{*}$, Louie Semaana, Adi L. Tarcab, Ma'In Yehya Awad Maitaha, Kentaro \\ HanadaC $^{\mathrm{C}}$, Jacek Bielawski ${ }^{\mathrm{d}}$, Maristella Villani ${ }^{\mathrm{d}}$, and Chiara Luberto $^{\mathrm{d}}$ \\ a Department of Pharmaceutical Sciences, Eugene Applebaum College of Pharmacy and Health Sciences, \\ Wayne State University, Detroit, MI 48201 \\ b Karmanos Cancer Institute, Wayne State University, Detroit, MI 48201
}

c Department of Biochemistry and Cell Biology, National Institute of Infectious Diseases, 1-23-1, Toyama, Shinjuku-ku, Tokyo 162-8640, Japan

d Department of Biochemistry and Molecular Biology, Medical University of South Carolina, Charleston, SC 29425

\begin{abstract}
We have shown that overexpression of SMS1, an enzyme that converts de novo ceramide into sphingomyelin, is accompanied by attenuated ceramide response and apoptotic resistance after photodamage with the photosensitizer Pc 4 (photodynamic therapy; PDT). To test whether SMS1 overexpression-related effects after PDT can be reversed, in this study SMS1 was downregulated in Jurkat T lymphoma/leukemia cells using small inhibitory RNA (siRNA) for SMS1. Compared to scrambled (control) siRNA-transfectants, in SMS1 siRNA-transfected cells the activity of SMS at rest was downregulated with concomitant decrease in sphingomyelin mass. In SMS1 siRNAtransfected cells increases in ceramides were higher than in control siRNA-transfectants after PDT. Similar findings were obtained for dihydroceramides suggesting the involvement of de novo ceramide pathway. PDT-induced DEVDase (caspase-3-like) activation was enhanced in SMS1 siRNA-transfected cells compared to their control counterparts. The data show that RNA interference-dependent downregulation of SMS1 is associated with increased accumulation of ceramide and dihydroceramide with concomitant sensitization of cells to apoptosis after photodamage. Similarly, in SMS2 siRNA-transfected cells, downregulation of SMS activity was accompanied by potentiated DEVDase activation post-photodamage. These findings suggest that SMS is a potential novel molecular target that can augment therapeutic efficacy of PDT.
\end{abstract}

\section{Keywords}

apoptosis; ceramide; dihydroceramide; electrospray ionization/double mass spectrometry; PDT; phthalocyanine Pc 4; sphingomyelin synthase

\footnotetext{
*Corresponding author. Department of Pharmaceutical Sciences, Eugene Applebaum College of Pharmacy and Health Sciences, Wayne State University, Detroit, MI 48201, USA. Fax: 313 577-2033. E-mail address: dseparovic@ wayne.edu (D. Separovic).

Publisher's Disclaimer: This is a PDF file of an unedited manuscript that has been accepted for publication. As a service to our customers we are providing this early version of the manuscript. The manuscript will undergo copyediting, typesetting, and review of the resulting proof before it is published in its final citable form. Please note that during the production process errors may be discovered which could affect the content, and all legal disclaimers that apply to the journal pertain.
} 


\section{Introduction}

Ceramide is a key metabolite in both anabolic and catabolic pathways of sphingolipids [1-4]. De novo sphingolipid biosynthesis begins with serine palmitoyltransferase (SPT)-dependent condensation of palmitoyl CoA and L-serine, resulting in the synthesis of 3-

ketodihydrosphingosine. In subsequent reactions dihydrosphingosine, dihydroceramide, and de novo ceramide are formed, and the latter can be acted upon by sphingomyelin synthase (SMS) to give rise to sphingomyelin. SMS1 and SMS2, two isoforms of the enzyme, are localized to the Golgi and plasma membrane, respectively [5]. Besides controlling cellular sphingomyelin and ceramide levels [6,7], SMS1 and SMS2 have been shown to regulate cell growth and apoptosis. RNA interference-induced suppression of SMS2 and/or SMS1 are associated with apoptotic resistance [8-10] and inhibition of growth [7].

However, de novo ceramide can be associated with apoptotic sensitization after oxidative stress [11]. The oxidative stress inducer photodynamic therapy uses a photosensitizer, visible light and oxygen to generate reactive oxygen species that can destroy malignant cells by apoptosis $[12,13]$. Using pharmacologic and genetic approaches, we have demonstrated that de novo sphingolipids are associated with initiation of apoptosis after photodamage with Pc 4 (PDT) $[14,15]$. We have shown that in the absence of SPT upregulation, de novo ceramide accumulates, while SMS is inhibited post-PDT [16]. These findings support the idea that PDT triggers de novo ceramide accumulation by inhibition of SMS. To test directly the role of SMS in ceramide production and apoptosis, we overexpressed SMS1 in Jurkat cells and found that both ceramide production and apoptosis are suppressed after PDT [17]. The aim of the present study was to test whether downregulation of SMS by siRNA can reverse the effects observed in SMS1-overexpressing cells and sensitize Jurkat cells to apoptosis post-PDT.

\section{Materials and methods}

\section{Cell culture}

Jurkat, clone E6-1 cells (American Type Culture Collection) were cultured in RPMI 1640 medium (Invitrogen), supplemented with 10\% fetal bovine serum (Hyclone), 100 units $/ \mathrm{ml}$ penicillin, and $100 \mu \mathrm{g} / \mathrm{ml}$ streptomycin, and were maintained at $37^{\circ} \mathrm{C}$ in a $5 \% \mathrm{CO}_{2}$ atmosphere. For PDT experiments, cells were treated in growth medium and all incubations were performed at $37^{\circ} \mathrm{C}$ in a $5 \% \mathrm{CO}_{2}$ atmosphere. The phthalocyanine photosensitizer Pc 4, HOSiPcOSi $\left(\mathrm{CH}_{3}\right)_{2}\left(\mathrm{CH}_{2}\right)_{3} \mathrm{~N}\left(\mathrm{CH}_{3}\right)_{2}$, was from Dr. Malcolm E. Kenney (Case Western Reserve University).

\section{Transfection with siRNA and treatment}

The sequences of siRNAs for human SMS1 and SMS2 were CAC ACT ATG GCC AAT CAG CAA and AAG GCA CCA AAA AGT ACC CGG, respectively. Two scrambled siRNA were initially tested: Silencer Negative Control \#1 (Ambion) and AllStars Negative Control siRNA (Qiagen). The latter was used for all studies described in this paper. Jurkat cells were transfected with double strand siRNAs by electroporation using the Amaxa Nucleofactor device (Amaxa) according to the manufacturer's instructions. The protocol was optimized regarding the concentration of SMS1 siRNA, transfection and post-transfection conditions in preliminary dose-response experiments (0.001- $3 \mu \mathrm{M}$ SMS1 siRNA), and using mock cells as controls. Consequently, the following protocol was employed: cells $\left(5 \times 10^{6}\right)$ were transfected with 1.5 $\mu \mathrm{M}$ of each siRNA. Two days after transfection, cells were collected and seeded in fresh growth medium containing Pc $4(200 \mathrm{nM})$. Following overnight exposure to Pc 4 , cells were irradiated with red light $\left(2 \mathrm{~mW} / \mathrm{cm}^{2} ; \lambda_{\max } \sim 670 \mathrm{~nm}\right)$ using a light-emitting diode array (EFOS) at various fluences $\left(135,270\right.$ and $\left.400 \mathrm{~mJ} / \mathrm{cm}^{2}\right)$ at room temperature. Two hours post-PDT, cells were harvested, washed with PBS, and further processed for various analyses. For mass spectrometric analysis, cells $\left(5 \times 10^{6}\right)$ were washed twice with PBS, resuspended in $100 \mu$ l ethyl 
acetate/methanol $(1: 1, \mathrm{v} / \mathrm{v})$, dried under nitrogen, and shipped overnight on dry ice to the Lipidomics Core (Charleston, SC) for further processing.

\section{Electrospray ionization/double mass spectrometry analysis}

Following extraction, sphingolipids were separated by high performance liquid chromatography, introduced to electrospray ionization source and then analyzed by double mass spectrometry (Thermo Finnigan TSQ 7000 triple quadrupole mass spectrometer), which allows simultaneous determination of various sphingolipids, including ceramide, dihydroceramide and sphingomyelin species, as well as dihydrosphingosine and sphingosine-1-phosphate [18]. Specifically, cells $\left(2-3 \times 10^{6}\right)$ were fortified with the internal standards (C17-base-D-erythro-sphingosine, C17-sphingosine-1-phosphate, N-palmitoyl-Derythro-C13-sphingosine, and C17-D-erythro-sphingosine), and extracted with ethyl acetate/ iso-propanol/water $(60 / 30 / 10, \mathrm{v} / \mathrm{v})$. After evaporation and reconstitution in $100 \mu \mathrm{l}$ of methanol, samples were injected into the HP1100/TSQ 7000 LC/MS system and gradient-eluted from the BDS Hypersil C8, $150 \times 3.2 \mathrm{~mm}, 3 \mu \mathrm{m}$ particle size column, with $1 \mathrm{mM}$ methanolic ammonium formate $/ 2 \mathrm{mM}$ aqueous ammonium formate mobile phase. Peaks corresponding to the target analytes and internal standards were collected and processed using the Xcalibur software system. Quantitative analysis was based on the calibration curves generated by spiking an artificial matrix with the known amounts of the target analyte synthetic standards and an equal amount of the internal standards. For the calibration curves, the target analyte/ internal standard peak area ratios were plotted against analyte concentration. The target analyte/ internal standard peak area ratios from the samples were similarly normalized to their respective internal standards and compared to the calibration curves, using a linear regression model. The method is referred to in the remaining text as mass spectrometry.

\section{SMS activity}

The enzyme activity was measured in cell lysates prepared from cells $\left(2.5 \times 10^{6}\right)$ treated with PDT as described previously $[19,20]$. The assay of SMS activity is based on the ability of SMS to incorporate the fluorogenic derivative of C6-ceramide, (N-[6-[(7-nitro-2-1,3benzoxadiazol-4-yl)amino]hexanoyl]-D-erythro-sphingosine (C6-NBD-ceramide; Avanti), into sphingomyelin. C6-NBD-ceramide-labeled sphingomyelin was extracted, separated by thin layer chromatography (TLC) using chloroform/methanol/water $(65 / 25 / 4, \mathrm{v} / \mathrm{v})$, and the fluorescence was detected and quantified by the STORM 860 imaging system (GE Healthcare).

\section{DEVDase (caspase-3-like) activity}

As described previously [16], the activity of the apoptotic marker DEVDase, was determined in cytosol by an assay based on the enzyme's ability to cleave the fluorogenic derivative 7amino-4-methylcoumarin (AMC; Biomol) of the tetrapeptide substrate N-acetyl-Asp-Glu-ValAsp (DEVD). The released fluorescence of the cleaved DEVD substrate was measured in a F-2500 Hitachi spectrofluorometer (380 nm excitation and $460 \mathrm{~nm}$ emission).

\section{Statistical analysis}

The -fold changes (ratios) of PDT-treated values divided by corresponding Pc 4 control value were $\log 2$ or $\log 10$ transformed to improve the normality of the distributions. The response variable Y (e.g., ceramide levels) was regressed (modeled) using linear mixed-effect models (LMEM) in which the dose and the group variables were considered as fixed effects (of interest) while the day variable (i.e., the day of experiment) was treated as a random effect (nuisance variable). The group variable was a factor with two levels (scrambled-, SMS1-transfected cells) and the dose was treated as a continuous variable. The main effects, i.e., that of the group and the dose, on the response $\mathrm{Y}$, were estimated along with their significance as predictors in the 
model. A separate model was fitted with the data for each sphingolipid molecule. The $\mathbf{R}$ statistical environment (www.r-project.org) was used to fit the LMEM model [21].

In the tables, the FCG is the estimated fold-change between groups (cell lines) regarding a response (e.g., ceramide level), assuming a constant dose. The FCD is the estimated foldchange in response to each 100 units of dose (light fluence), regardless of the group. The pGroupFDR and the pDoseFDR are the corresponding adjusted p-values using a false discovery rate adjustment algorithm [22]. This adjustment is required to account for multiple testing (i.e. several molecules tested at the same time) and therefore controls the fraction of false positive findings among all positive test results. For example, if 20 ceramides are tested at the same time and 10 of them had pGroupFDR less than 0.05 , this means about $0.05 * 10=$ 0.5 of them (less than 1) might be false positives, which is an acceptable risk. The significance of the group coefficient in the linear model is tested assuming a T distribution, and it is implicitly a two-tailed test.

\section{Results}

To verify downregulation of SMS1 by RNA interference in Jurkat cells, the activity of the enzyme was determined in cell lysates (Fig. 1) and the levels of sphingomyelin, a product of SMS, were measured by mass spectrometry (Table 1). As shown in Figure 1, the enzyme activity was downregulated in SMS1 siRNA-transfectants. Based on the calculated FCG value of 0.65 , the enzyme activity was reduced by $54 \%$ in SMS1 siRNA-transfectants. [The formula used for calculating percent reduction here and elsewhere was: $(1 / \mathrm{FCG}-1)^{*} 100$.] Using mass spectrometry we found that the levels of eight out of twelve sphingomyelin species were significantly reduced in SMS1 siRNA-transfectants (Table 1). C14-, C16-, C18-, C20-, C22-, C22:1-, C24-, C24:1-sphingomyelin were reduced in SMS1 siRNA-transfectants by 39, 55, $51,70,59,66,58$, and $39 \%$, respectively. The trends for remaining four sphingomyelins were similar but not significant (Table 1).

We used mass spectrometry to compare the effects of photodamage in SMS1 siRNAtransfectants and scrambled siRNA-transfectants on the mass of sphingolipids. Twelve species were detected for each of the following sphingolipids: ceramides, dihydroceramides and sphingomyelins. Among ceramides, all but C24- and C24:1-ceramide showed a dosedependent response to PDT in both cell types, as indicated by pDoseFDRs (Table 2). C22:1ceramide showed maximal dose-response since the calculated FCD value of 2.02 indicates an overall 102\% increase in C22:1-ceramide in both cell types per 100 units of light fluence (Table 2). Five out of twelve ceramides showed significantly enhanced response to PDT in SMS1 siRNA-transfectants compared to scrambled siRNA-transfectants (see pGroupFDRs, Table 2; Fig. 2). Specifically, compared to their control counterparts, in SMS1 siRNA-transfected cells after PDT there was on average a 74, 35, 36, 56 and 45\% greater response of C26-, C24-, C24:1-, C22- and C26:1-ceramide, respectively.

Dihydroceramides, which are precursors of ceramide in the de novo pathway, were also detected by mass spectrometry. All twelve dihydroceramides showed a dose-dependent response to PDT in both cell types (see pDoseFDRs, Table 2). C18-dihydroceramide showed maximal dose response since the calculated FCD value of 3.98 indicates an overall $298 \%$ increase in C18-dihydroceramide in both cell types per 100 units of light fluence (Table 2). Three out of twelve dihydroceramides showed significantly enhanced response to PDT in SMS1 siRNA-transfectants relative to scrambled siRNA-transfectants (see pGroupFDRs, Table 2; Fig. 3). Compared to scrambled siRNA-transfected cells, in SMS1 siRNAtransfectants after PDT there was on average a 67, 40, and 27\% greater response of C24:1-, C24- and C22-dihydroceramide, respectively. 
None of the twelve sphingomyelins showed a dose-dependent response to PDT in either cell type (see pDoseFDRs, Table 2). FCD values indicate that the overall sphingomyelin levels were close to resting levels in both cell types after PDT. All but C14-sphingomyelin showed no difference between two cell types (see pGroupFDRs, Table 2). Downregulation of SMS1 was associated with a 33\% greater response of C14-sphingomyelin to PDT, resulting in closer to resting sphingomyelin levels.

Dihydrosphingosine, another precursor of ceramide in the de novo pathway, was also detected by mass spectrometry. As indicated by the pDoseFDR, the sphingolipid showed a dosedependent response to PDT in both cell types (Table 2). The calculated FCD value indicates an overall $64 \%$ increase in dihydrosphingosine per 100 units of light fluence in either cell type (Table 2). However, there was no difference between the two cell types in the response of this sphingolipid to PDT (Table 2).

The accumulation of the proapoptotic sphingosine and the antiapoptotic sphingosine-1phosphate after PDT showed significant differences between the two cell types (Table 2; Fig. 4). Generation of sphingosine was $135 \%$ greater in SMS1 siRNA-transfectants compared to scrambled siRNA-transfectants (Fig. 4A). In contrast, the levels of sphingosine-1-phosphate were 87\% lower in SMS1 siRNA-transfectants than in their control counterparts (Fig. 4B). A significant dose-response was detected only for sphingosine-1-phosphate. The calculated FCD value of 1.28 indicates an overall 28\% increase in sphingosine-1-phosphate per 100 units of light fluence in either cell type (Table 2). Dihydrosphingosine-1-phosphate, which is, in addition to sphingosine-1-phosphate, a product of sphingosine kinase, showed a dose-response in both cell types but no difference between them after photodamage (Table 2).

We have shown that PDT triggers inhibition of SMS without affecting SPT activity in Jurkat parental cells [16]. The activity of SMS was determined after PDT in SMS1 siRNAtransfectants and their control counterparts. The calculated FCD value of 0.53 shows an overall significant $90 \%$ decrease in the enzyme activity per 100 units of light fluence in both cell types (not shown). However, no significant difference between the two cell types was detected after photodamage (Figure 5A).

We have already shown that Jurkat cells undergo apoptosis post-PDT [15,23], and that overexpression of SMS1 leads to resistance to PDT [17]. In this study we tested whether Jurkat cells can be sensitized to PDT by downregulation of SMS1. Apoptosis was assessed by measuring DEVDase (caspase-3-like) activity. Activation of caspase-3 is involved in the execution of apoptosis [24]. The calculated FCD value of 1.94 shows a $94 \%$ increase in the enzyme activity per 100 units of light fluence in both cell types (Table 3). Notably, DEVDase activity was potentiated by $65 \%$ post-PDT in SMS1 siRNA-transfectants compared to their control counterparts (Table 3; Fig. 5B). The data demonstrate that PDT initiates a dosedependent DEVDase activation in both cell types, and that compared to scrambled siRNAtransfectants the responses were substantially enhanced in SMS1 siRNA-transfectants.

Similar experiments were performed in SMS2 siRNA-transfectants. As shown in Figure 6A, the enzyme activity was downregulated in SMS2 siRNA-transfectants. Based on the calculated FCG value of 0.67 (not shown), the enzyme activity was reduced by 49\% in SMS2 siRNAtransfectants. For DEVDase, there was an overall $61 \%$ increase in the enzyme activity per 100 units of light fluence in both cell types (Table 3). Importantly, DEVDase activity was enhanced by 53\% post-PDT in SMS2 siRNA-transfectants compared to their controls (Table 3; Fig. 6B). The data show that, PDT triggered a dose-dependent DEVDase activation in both cell types, and that compared to scrambled siRNA-transfectants the response was promoted in SMS2 siRNA-transfectants. 


\section{Discussion}

The present study shows for the first time that downregulation of SMS1 is associated with enhanced accumulation of proapoptotic sphingolipids and promotion of apoptosis after PDT. The correlation between PDT, proapoptotic sphingolipids, and apoptosis is further supported by the evidence that all twelve dihydroceramides, ten out of twelve ceramides, and sphingosine showed a dose-dependent increase to PDT. Moreover, the present study indicates that the sphingolipid balance is reset by SMS1 downregulation in favor of the enhanced accumulation of proapoptotic ceramide, dihydroceramide and sphingosine, and the decrease in the antiapoptotic sphingosine-1-phosphate, with concomitant promotion of apoptosis post-PDT. The cellular balance between proapoptotic ceramide and antiapoptotic sphingosine-1phosphate has been proposed to be critical for the life or death of the cell [25].

Three long-chain dihydroceramides (i.e., C22-, C24-, and C24:1-dihydroceramide) and five long-fatty acyl chain ceramides (i.e., C22-, C24-, C24:1-, C26-, and C26:1-ceramide) show enhanced increase to PDT in cells with downregulated SMS1, suggesting potential selectivity. Similarly, C24-ceramide has been associated with apoptosis [26]. The biological significance of specific fatty acyl chains in ceramides remains to be established.

Besides dihydroceramides, PDT also induced a dose-dependent increase in dihydrosphingosine, another precursor from the de novo sphingolipid biosynthesis. The data are consistent with the notion that the de novo sphingolipid pathway is a PDT target. The idea is supported by our previously published evidence [14-16,23].

A dose-dependent increase of sphingosine-1-phosphate, a product of sphingosine kinase, was triggered by PDT. It is unlikely that sphingosine kinase is a target of the primary photodamage since the enzyme is primarily found in the cytosol [27], where Pc 4 is not found. Pc 4 localizes to intracellular membranes, e.g., mitochondria, the ER and Golgi, but not the plasma membrane or nuclear membrane [28]. The primary photodamage, most likely via singlet oxygen, occurs where Pc 4 is localized. This is so because singlet oxygen has a short lifetime $(<0.04 \mu \mathrm{s})$ and, consequently, a short radius of action $(0.02 \mu \mathrm{m})$ [29], and it will remain at the site of origin, e.g., in the Golgi. If there is an effect of PDT on sphingosine kinase, it could be a secondary effect, e.g., from the oxidative damage to mitochondria with subsequent inhibition of electron transport [30-32] and generation of superoxide. Although there is evidence to the contrary [33], superoxide can diffuse from mitochondria to cytosol [34], and presumably affect sphingosine kinase. Incidentally, we have shown that manganese superoxide dismutase, the scavenger of superoxide produced by mitochondrial electron transport, affects sensitivity of cells to PDT, suggesting the involvement of superoxide [23].

Previously we proposed that, in the absence of SPT activation, inhibition of SMS is associated with increased accumulation of de novo ceramide [16]. In this study we show that SMS1 downregulation was accompanied by increased accumulation of ceramides and dihydroceramides without a change in the extent of inhibition of SMS by PDT. In addition, there was a lack of correlation between SMS activity and sphingomyelins in both cell types. Besides SMS, sphingomyelinase [1] and ethanolamine phosphorylceramide synthase [35] can be involved in regulation of sphingomyelin levels. Overall, the data suggest that following photodamage in the cells with downregulated SMS1, the activities of enzymes other than SMS are affected.

We have shown that SMS1-overexpressing cells are resistant to apoptosis after PDT [17]. Here we demonstrate that downregulation of SMS1, as well as SMS2, correlates with apoptotic sensitization to PDT. In agreement with our findings, others have shown a correlation between apoptotic resistance and increased SMS activity [36,37]. However, there is evidence to the contrary, i.e., siRNA interference-dependent downregulation of SMS1 is accompanied by 
apoptotic resistance [8-10]. The discrepancy regarding the role of SMS in apoptosis could be due to different stimuli and/or different cell types used in these studies, with potentially different underlying mechanisms. Similarly, we have shown that the effects of PDT on de novo ceramide metabolism could not be completely reproduced either by another proapoptotic agent in the same cell type [38] or by PDT itself in two different cell types [16]. Regardless, our findings imply that both SMS1 and SMS2 are PDT molecular targets with a potential of regulating apoptosis.

\section{Acknowledgements}

This work was supported by U.S. Public Health Service Grant R01 CA77475 from the National Cancer Institute, National Institutes of Health. The mass spectroscopy-related work was done at the Lipidomics Core (Department of Biochemistry and Molecular Biology, Medical University of South Carolina, Charleston), a facility supported by the U.S. Public Health Service Grant C06 RR018823 from the Extramural Research Facilities Program, National Center for Research Resources.

\section{References}

1. Hannun YA, Luberto C, Argraves KM. Enzymes of sphingolipid metabolism: from modular to integrative signaling. Biochemistry 2001;40:4893-4903. [PubMed: 11305904]

2. Hanada K. Serine palmitoyltransferase, a key enzyme of sphingolipid metabolism. Biochim Biophys Acta 2003;1632:16-30. [PubMed: 12782147]

3. Kester M, Kolesnick R. Sphingolipids as therapeutics. Pharmacol Res 2003;47:365-371. [PubMed: 12676510]

4. Zheng W, Kollmeyer J, Symolon H, Momin A, Munter E, Wang E, Kelly S, Allegood JC, Liu Y, Peng Q, Ramaraju H, Sullards MC, Cabot M, Merrill AH Jr. Ceramides and other bioactive sphingolipid backbones in health and disease: lipidomic analysis, metabolism and roles in membrane structure, dynamics, signaling and autophagy. Biochim Biophys Acta 2006;1758:1864-1884. [PubMed: 17052686]

5. Huitema K, Van Den Dikkenberg J, Brouwers JF, Holthuis JC. Identification of a family of animal sphingomyelin synthases. Embo J 2004;23:33-44. [PubMed: 14685263]

6. Li Z, Hailemariam TK, Zhou H, Li Y, Duckworth DC, Peake DA, Zhang Y, Kuo MS, Cao G, Jiang XC. Inhibition of sphingomyelin synthase (SMS) affects intracellular sphingomyelin accumulation and plasma membrane lipid organization. Biochim Biophys Acta 2007;1771:1186-1194. [PubMed: 17616479]

7. Tafesse FG, Huitema K, Hermansson M, van der Poel S, van den Dikkenberg J, Uphoff A, Somerharju P, Holthuis JC. Both sphingomyelin synthases SMS1 and SMS2 are required for sphingomyelin homeostasis and growth in human HeLa cells. J Biol Chem 2007;282:17537-17547. [PubMed: 17449912]

8. Ding T, Li Z, Hailemariam T, Mukherjee S, Maxfield FR, Wu MP, Jiang XC. SMS overexpression and knockdown: impact on cellular sphingomyelin and diacylglycerol metabolism, and cell apoptosis. J Lipid Res 2008;49:376-385. [PubMed: 17982138]

9. Van der Luit AH, Budde M, Zerp S, Caan W, Klarenbeek JB, Verheij M, Van Blitterswijk WJ. Resistance to alkyl-lysophospholipid-induced apoptosis due to downregulated sphingomyelin synthase 1 expression with consequent sphingomyelin-and cholesterol-deficiency in lipid rafts. Biochem J 2007;401:541-549. [PubMed: 17049047]

10. van der Luit AH, Vink SR, Klarenbeek JB, Perrissoud D, Solary E, Verheij M, van Blitterswijk WJ. A new class of anticancer alkylphospholipids uses lipid rafts as membrane gateways to induce apoptosis in lymphoma cells. Mol Cancer Ther 2007;6:2337-2345. [PubMed: 17699729]

11. Carracedo A, Geelen MJ, Diez M, Hanada K, Guzman M, Velasco G. Ceramide sensitizes astrocytes to oxidative stress: protective role of cannabinoids. Biochem J 2004;380:435-440. [PubMed: 14979873]

12. Castano AP, Mroz P, Hamblin MR. Photodynamic therapy and anti-tumour immunity. Nat Rev Cancer 2006;6:535-545. [PubMed: 16794636]

13. Miller JD, Baron ED, Scull H, Hsia A, Berlin JC, McCormick T, Colussi V, Kenney ME, Cooper KD, Oleinick NL. Photodynamic therapy with the phthalocyanine photosensitizer Pc 4: the case 
experience with preclinical mechanistic and early clinical-translational studies. Toxicol Appl Pharmacol 2007;224:290-299. [PubMed: 17397888]

14. Wispriyono B, Schmelz E, Pelayo H, Hanada K, Separovic D. A role for the de novo sphingolipids in apoptosis of photosensitized cells. Exp Cell Res 2002;279:153-165. [PubMed: 12213223]

15. Dolgachev V, Nagy B, Taffe B, Hanada K, Separovic D. Reactive oxygen species generation is independent of de novo sphingolipids in apoptotic photosensitized cells. Exp Cell Res 2003;288:425436. [PubMed: 12915133]

16. Dolgachev V, Farooqui MS, Kulaeva OI, Tainsky MA, Nagy B, Hanada K, Separovic D. De novo ceramide accumulation due to inhibition of its conversion to complex sphingolipids in apoptotic photosensitized cells. J Biol Chem 2004;279:23238-23249. [PubMed: 15020599]

17. Separovic D, Hanada K, Maitah MY, Nagy B, Hang I, Tainsky MA, Kraniak JM, Bielawski J. Sphingomyelin synthase 1 suppresses ceramide production and apoptosis post-photodamage. Biochem Biophys Res Commun 2007;358:196-202. [PubMed: 17467659]

18. Bielawski J, Szulc ZM, Hannun YA, Bielawska A. Simultaneous quantitative analysis of bioactive sphingolipids by high-performance liquid chromatography-tandem mass spectrometry. Methods 2006;39:82-91. [PubMed: 16828308]

19. Futerman AH, Stieger B, Hubbard AL, Pagano RE. Sphingomyelin synthesis in rat liver occurs predominantly at the cis and medial cisternae of the Golgi apparatus. J Biol Chem 1990;265:86508657. [PubMed: 2187869]

20. Luberto C, Toffaletti DL, Wills EA, Tucker SC, Casadevall A, Perfect JR, Hannun YA, Del Poeta M. Roles for inositol-phosphoryl ceramide synthase 1 (IPC1) in pathogenesis of C. neoformans. Genes Dev 2001;15:201-212. [PubMed: 11157776]

21. Chambers, JM.; Hastie, TJ. Statistical Models. S, Wadsworth \& Brooks/Cole; Pacific Grove: 1992.

22. Benjamini Y, Hochberg Y. Controlling the false discovery rate: a practical and powerful approach to multiple testing. J Royal Stat Soc B 1995;57:289-300.

23. Dolgachev V, Oberley LW, Huang TT, Kraniak JM, Tainsky MA, Hanada K, Separovic D. A role for manganese superoxide dismutase in apoptosis after photosensitization. Biochem Biophys Res Commun 2005;332:411-417. [PubMed: 15894290]

24. Hengartner MO. The biochemistry of apoptosis. Nature 2000;407:770-776. [PubMed: 11048727]

25. Spiegel S, Milstien S. Sphingosine-1-phosphate: signaling inside and out. FEBS Lett 2000;476:5557. [PubMed: 10878250]

26. Kroesen BJ, Jacobs S, Pettus BJ, Sietsma H, Kok JW, Hannun YA, de Leij LF. BcR-induced apoptosis involves differential regulation of $\mathrm{C} 16$ and $\mathrm{C} 24$-ceramide formation and sphingolipid-dependent activation of the proteasome. J Biol Chem 2003;278:14723-14731. [PubMed: 12578840]

27. Pyne S, Pyne NJ. Sphingosine 1-phosphate signalling in mammalian cells. Biochem J 2000;349:385402. [PubMed: 10880336]

28. Lam M, Oleinick NL, Nieminen AL. Photodynamic therapy-induced apoptosis in epidermoid carcinoma cells. Reactive oxygen species and mitochondrial inner membrane permeabilization. J Biol Chem 2001;276:47379-47386. [PubMed: 11579101]

29. Moan J, Berg K. The photodegradation of porphyrins in cells can be used to estimate the lifetime of singlet oxygen. Photochem Photobiol 1991;53:549-553. [PubMed: 1830395]

30. Gibson SL, Hilf R. Interdependence of fluence, drug dose and oxygen on hematoporphyrin derivative induced photosensitization of tumor mitochondria. Photochem Photobiol 1985;42:367-373. [PubMed: 3003767]

31. Musser DA, Oseroff AR. The use of tetrazolium salts to determine sites of damage to the mitochondrial electron transport chain in intact cells following in vitro photodynamic therapy with Photofrin II. Photochem Photobiol 1994;59:621-626. [PubMed: 8066121]

32. Varnes ME, Chiu SM, Xue LY, Oleinick NL. Photodynamic therapy-induced apoptosis in lymphoma cells: translocation of cytochrome $c$ causes inhibition of respiration as well as caspase activation. Biochem Biophys Res Commun 1999;255:673-679. [PubMed: 10049769]

33. Missirlis F, Hu J, Kirby K, Hilliker AJ, Rouault TA, Phillips JP. Compartment-specific protection of iron-sulfur proteins by superoxide dismutase. J Biol Chem 2003;278:47365-47369. [PubMed: 12972424] 
34. Han D, Antunes F, Canali R, Rettori D, Cadenas E. Voltage-dependent anion channels control the release of the superoxide anion from mitochondria to cytosol. J Biol Chem 2003;278:5557-5563. [PubMed: 12482755]

35. Malgat M, Maurice A, Baraud J. Sidedness of ceramide-phosphoethanolamine synthesis on rat liver and brain microsomal membranes. J Lipid Res 1987;28:138-143. [PubMed: 3033117]

36. Itoh M, Kitano T, Watanabe M, Kondo T, Yabu T, Taguchi Y, Iwai K, Tashima M, Uchiyama T, Okazaki T. Possible role of ceramide as an indicator of chemoresistance: decrease of the ceramide content via activation of glucosylceramide synthase and sphingomyelin synthase in chemoresistant leukemia. Clin Cancer Res 2003;9:415-423. [PubMed: 12538495]

37. Yang Z, Khoury C, Jean-Baptiste G, Greenwood MT. Identification of mouse sphingomyelin synthase 1 as a suppressor of Bax-mediated cell death in yeast. FEMS Yeast Res 2006;6:751-762. [PubMed: 16879426]

38. Separovic D, Wang S, Awad Maitah MY, Hanada K, Kessel D. Ceramide response post-photodamage is absent after treatment with HA14-1. Biochem Biophys Res Commun 2006;345:803-808.

[PubMed: 16701558] 


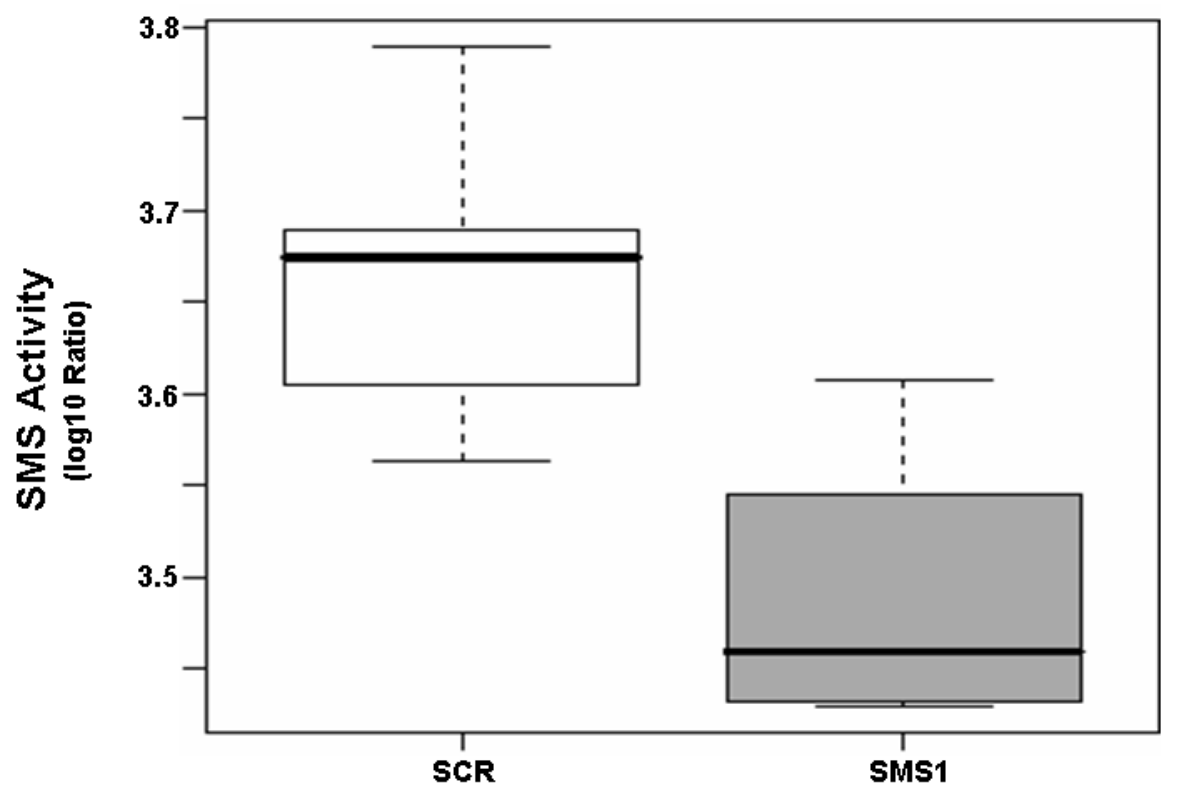

Figure 1.

Sphingomyelin synthase (SMS) is downregulated in SMS1 siRNA-transfected Jurkat cells. Cells were transfected with SMS1- or scrambled siRNA as described in "Material and Methods". Two days after transfection, cells were collected and seeded in fresh growth medium with or without Pc 4 (200 nM). Following overnight exposure to Pc 4, cells were harvested, and the enzyme activity was determined in cell lysates as described in "Material and Methods". There was no significant difference between Pc 4- and untreated-controls in either cell type, and for the analysis, the values of the two controls were grouped. SCR, scrambled siRNAtransfectants; SMS1, SMS1 siRNA -transfectants. 

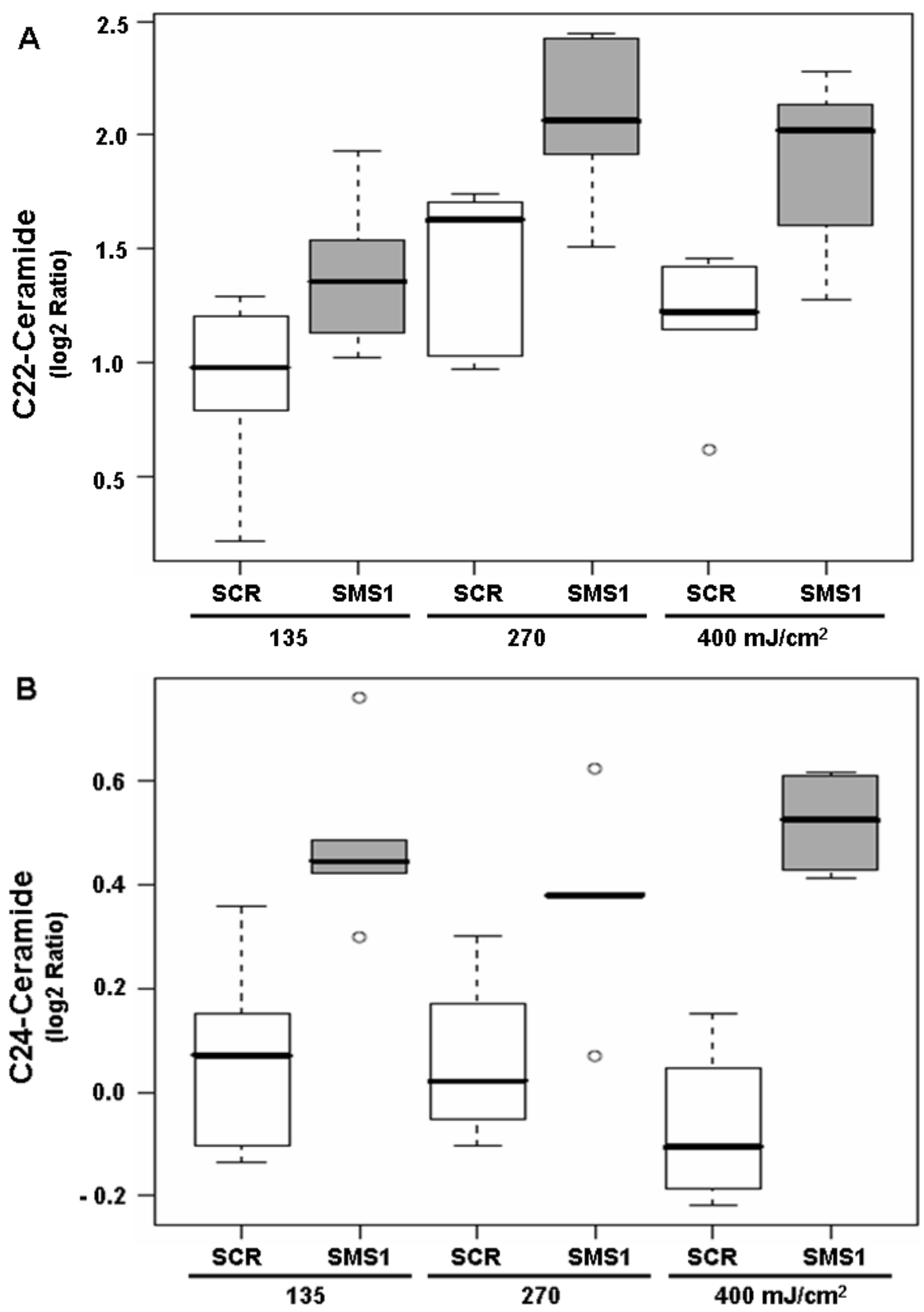

Exp Cell Res. Author manuscript; available in PMC 2009 May 1. 

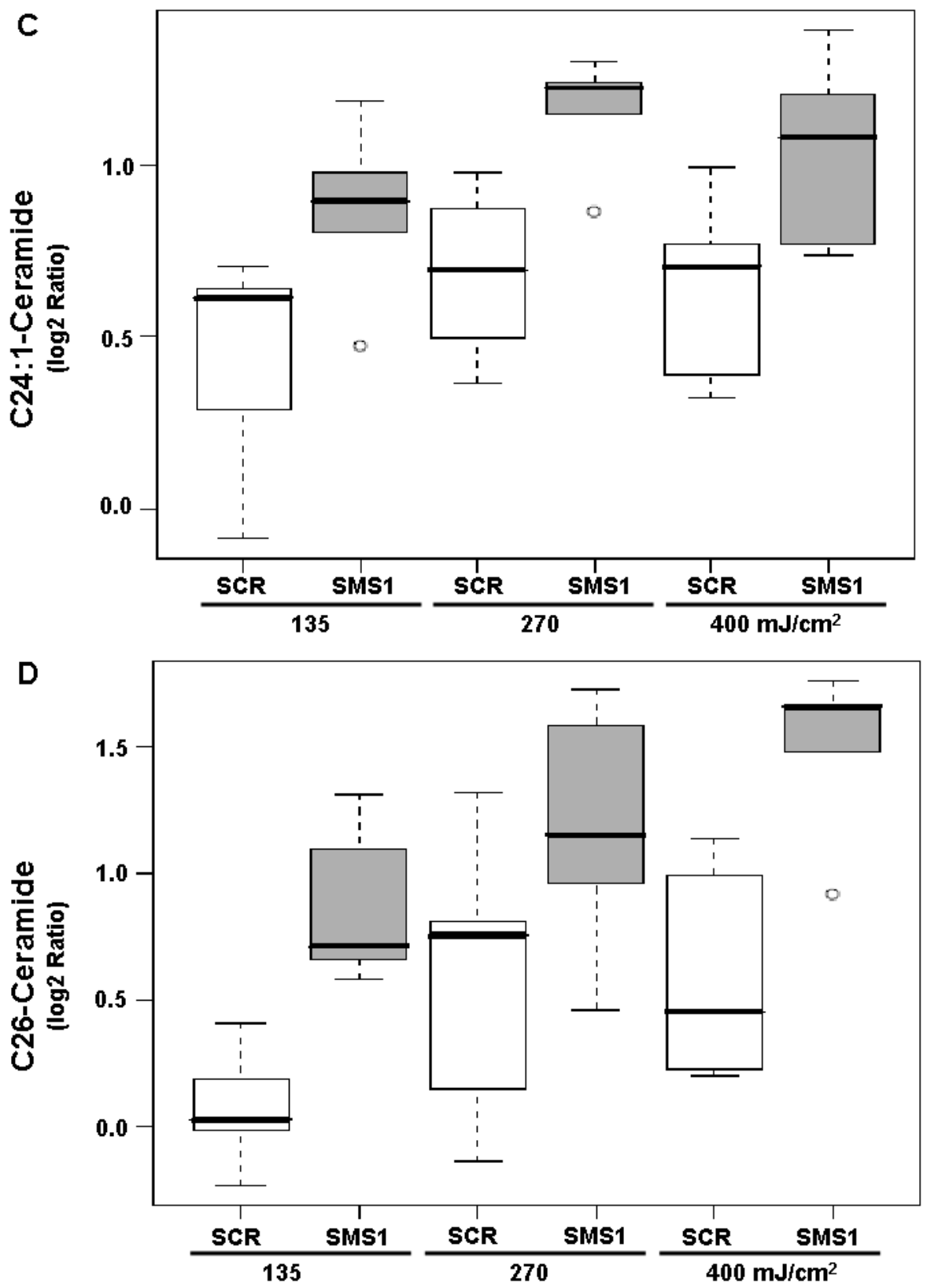

Exp Cell Res. Author manuscript; available in PMC 2009 May 1. 


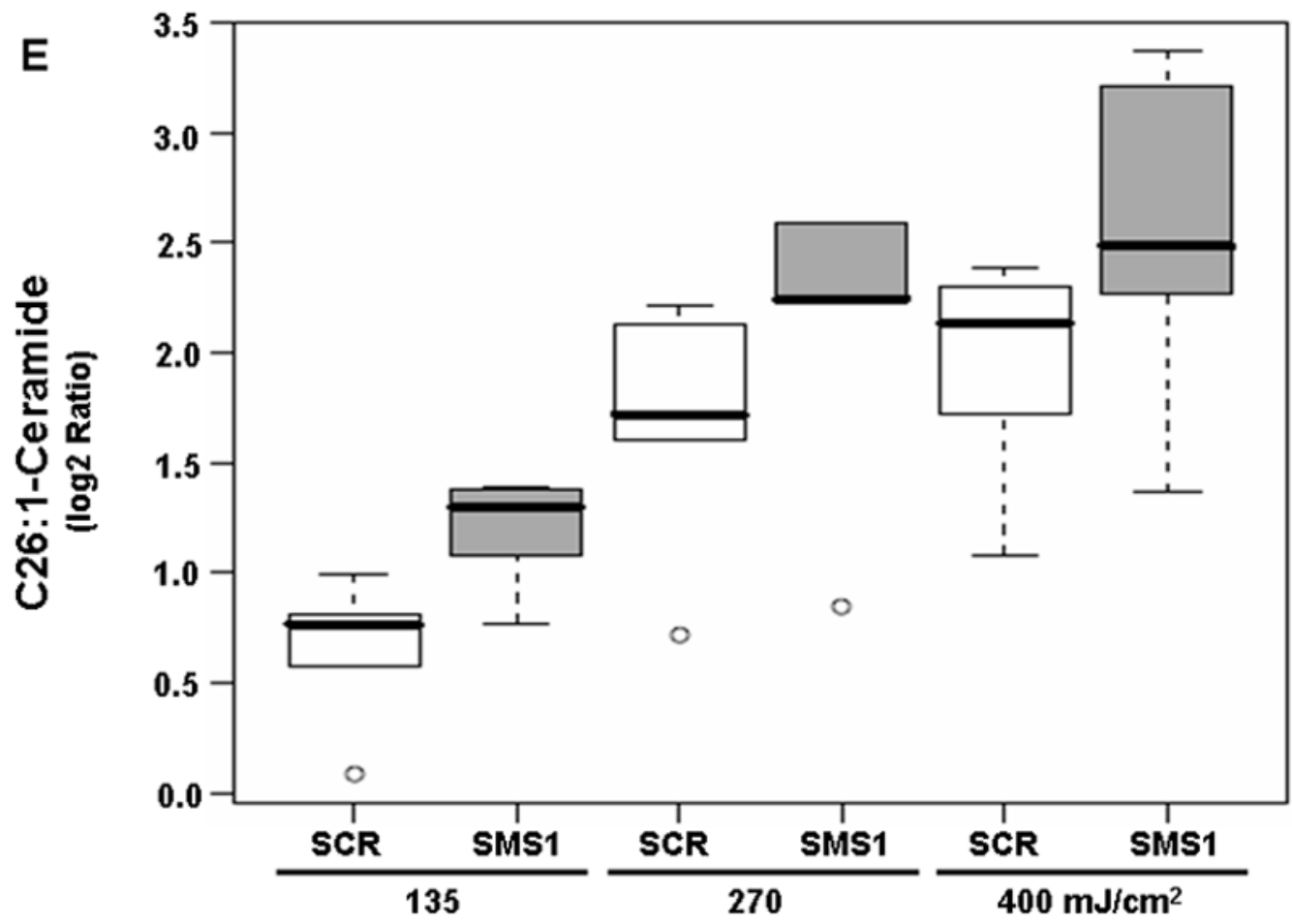

Figure 2.

Mass spectrometric analysis reveals enhanced ceramide response to PDT in SMS1 siRNAtransfected Jurkat cells. (A) C22-ceramide; (B) C24-ceramide; (C) C24:1-ceramide; (D) C26ceramide; (E) C26:1-ceramide. Cells were transfected with SMS1- or scrambled-siRNA as described in "Materials and Methods". Two days after transfection, cells were collected and seeded in fresh growth medium with or without Pc 4 (200 nM). Following overnight exposure to Pc 4, cells were irradiated with red light at the indicated light fluences $\left(\mathrm{mJ} / \mathrm{cm}^{2}\right)$. Two hours post-PDT cells were harvested, and lipids were extracted. Ceramide levels were determined by mass spectrometry (see "Materials and Methods"). Three-six independent measurements of ceramides were performed. The $\mathrm{Y}$-axis shows $\log 2$ ratios for each ceramide as a function of both group and dose variable. This and subsequent figures: the boxes contain $50 \%$ of the data and the median value is shown as the horizontal thick line within the box. The whiskers extend to the most extreme data point (minimum and maximum value) which is no more than 1.5 times the interquartile range (i.e., remaining $50 \%$ of the data) from the box. Outliers are shown as empty circles. SCR, scrambled siRNA-transfectants; SMS1, SMS1 siRNA transfectants. 

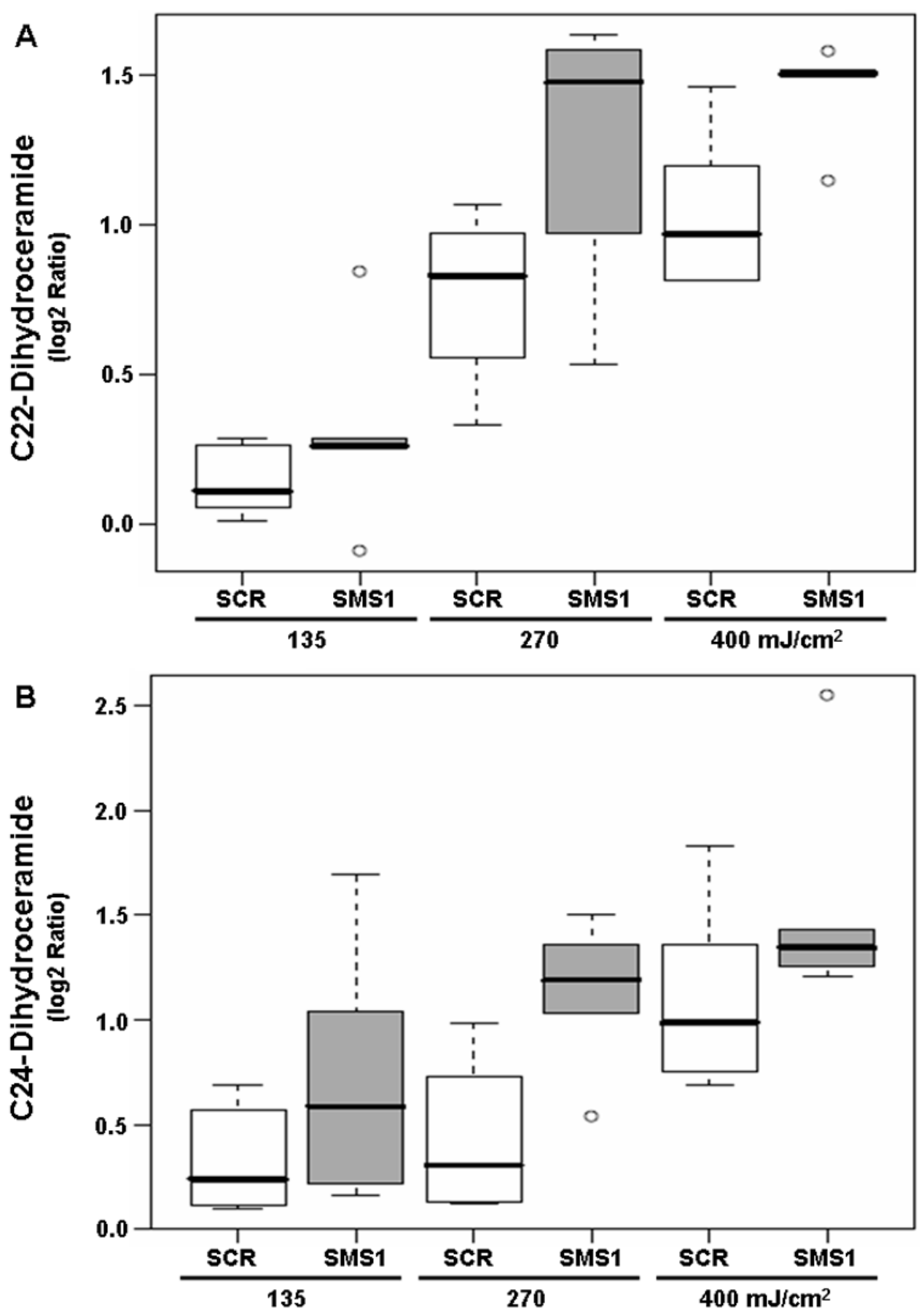

Exp Cell Res. Author manuscript; available in PMC 2009 May 1. 


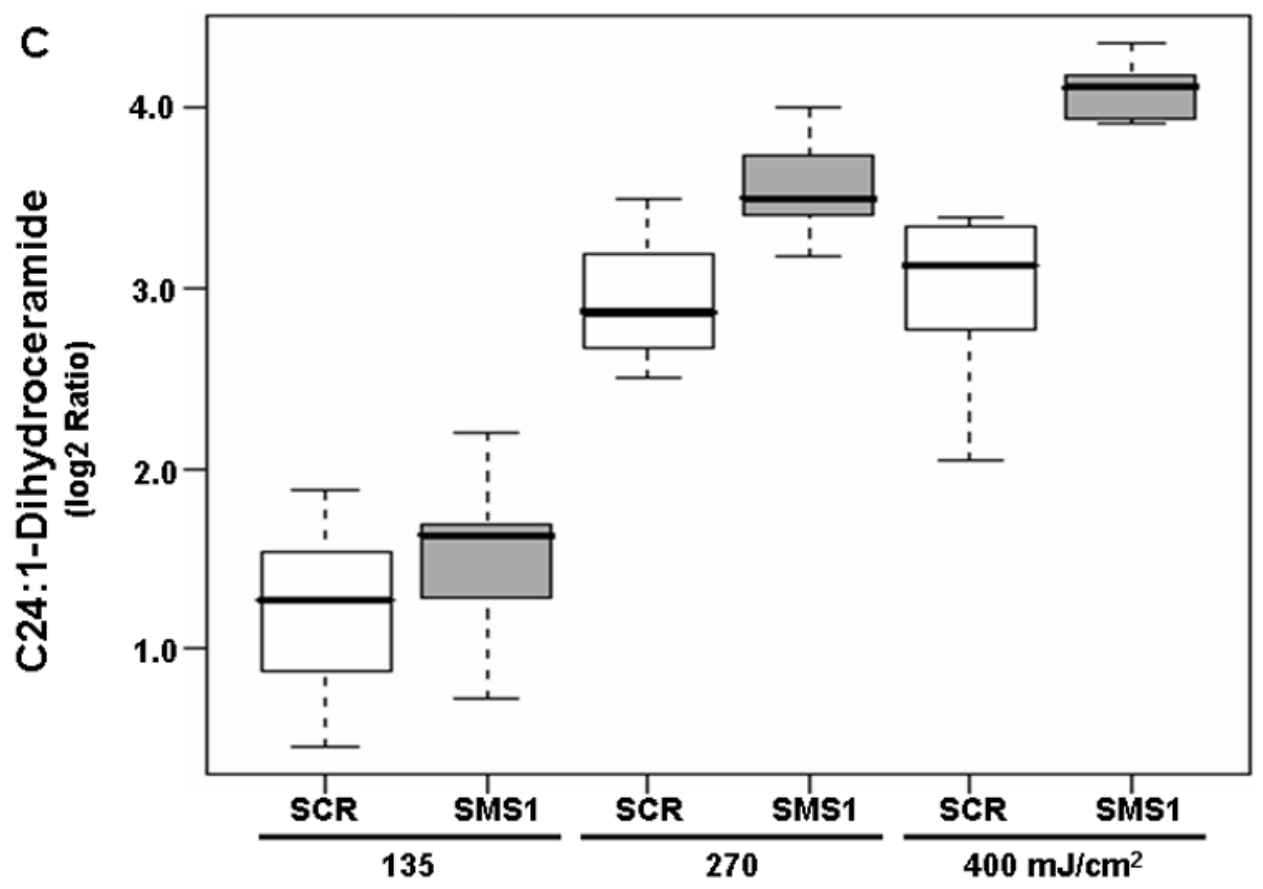

Figure 3.

Mass spectrometric analysis reveals enhanced dihydroceramide response to PDT in siRNA SMS1-transfected Jurkat cells. (A) C22-dihydroceramide; (B) C24-dihydroceramide; (C) C24:1-dihydroceramide. See Fig. 2 for experimental details. Dihydroceramide levels were determined by mass spectrometry (see "Materials and Methods"). Three-six independent measurements of ceramides were performed. The Y-axis shows $\log 2$ ratios for each ceramide as a function of both group and dose variable. Outliers are shown as empty circles. SCR, scrambled siRNA-transfectants; SMS1, SMS1 siRNA -transfectants. 

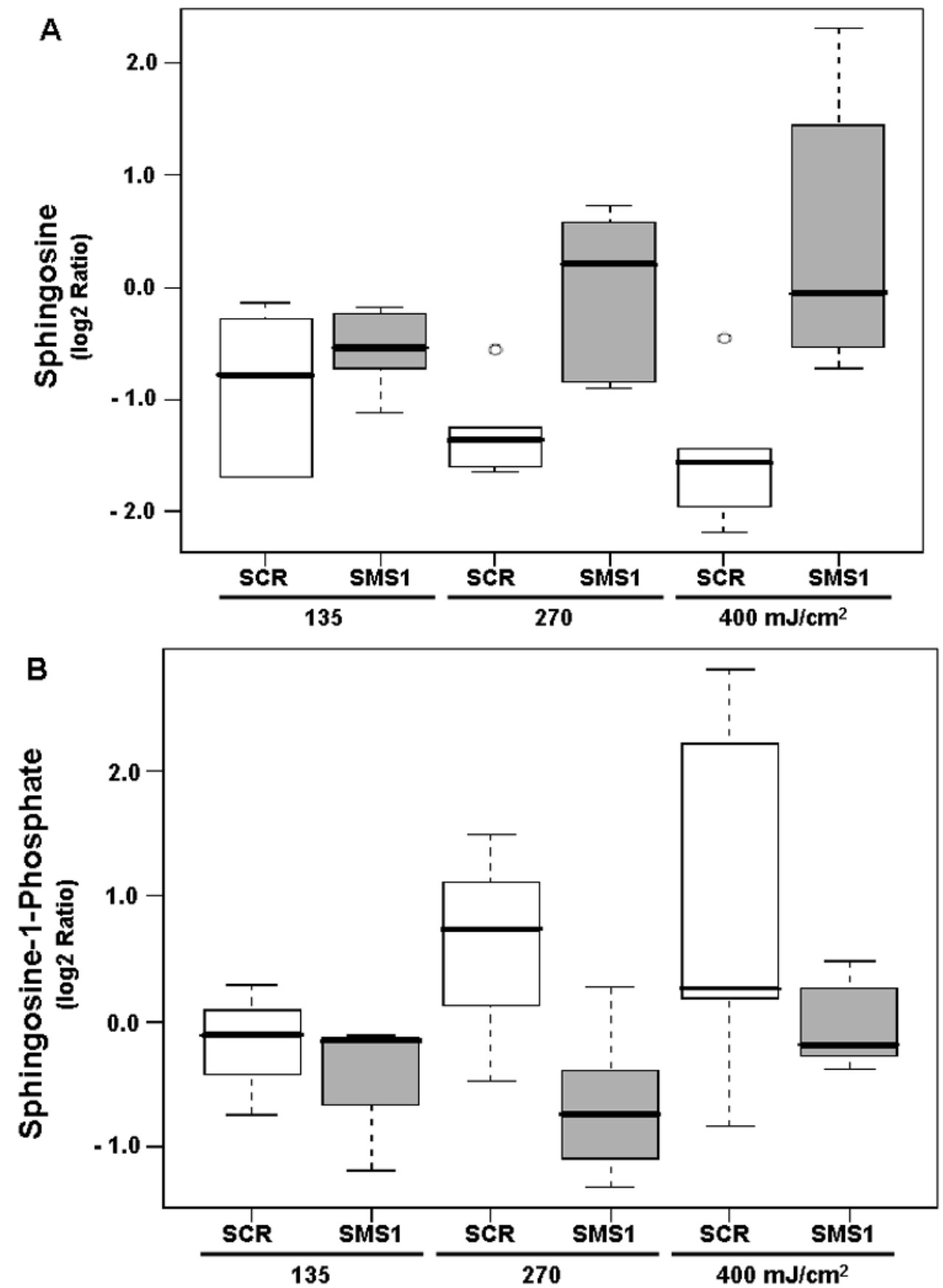

Figure 4.

Mass spectrometric analysis identifies different responses of sphingosine (A) and sphingosine-1-phosphate (B) to PDT in SMS1 siRNA-transfected Jurkat cells compared to their control counterparts. See Fig. 2 for experimental details. Sphingolipid levels were determined by mass spectrometry (see "Materials and Methods"). Three-six independent measurements of sphingolipids were performed. The Y-axis shows $\log 2$ ratios for each sphingolipid as a function of both group and dose variable. Outliers are shown as empty circles. SCR, scrambled siRNA-transfectants; SMS1, SMS1 siRNA -transfectants. 

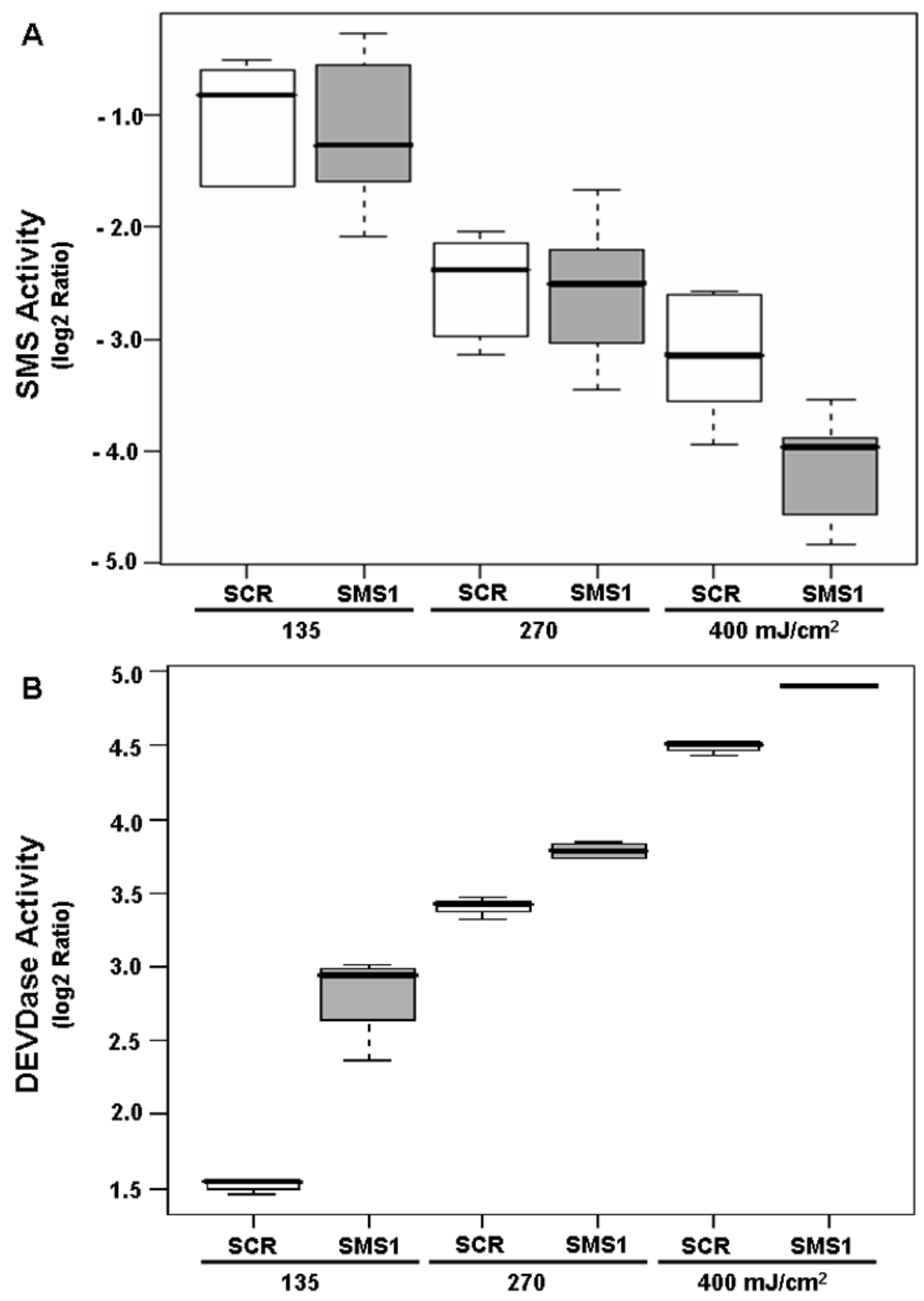

Figure 5.

The effects of PDT on the activities of SMS (A) and DEVDase (B) in scrambled siRNA- and SMS1 siRNA-transfected Jurkat cells. Forty eight hours after transfection, cells were treated overnight with Pc 4 (200 nM), irradiated at the indicated light fluences, and incubated for $2 \mathrm{~h}$. After the cells were harvested, (A) cell lysates were prepared and the activity of SMS was assayed using C6-NBD-ceramide as the substrate; (B) DEVDase activity was measured in cell lysates spectrofluorimetrically using DEVD-AMC as the substrate. Three-five independent determinations of the enzymes were performed. The Y-axis shows $\log 2$ ratios for each enzyme as a function of both group and dose variable. SCR, scrambled siRNA-transfectants; SMS1, SMS1 siRNA -transfectants. 

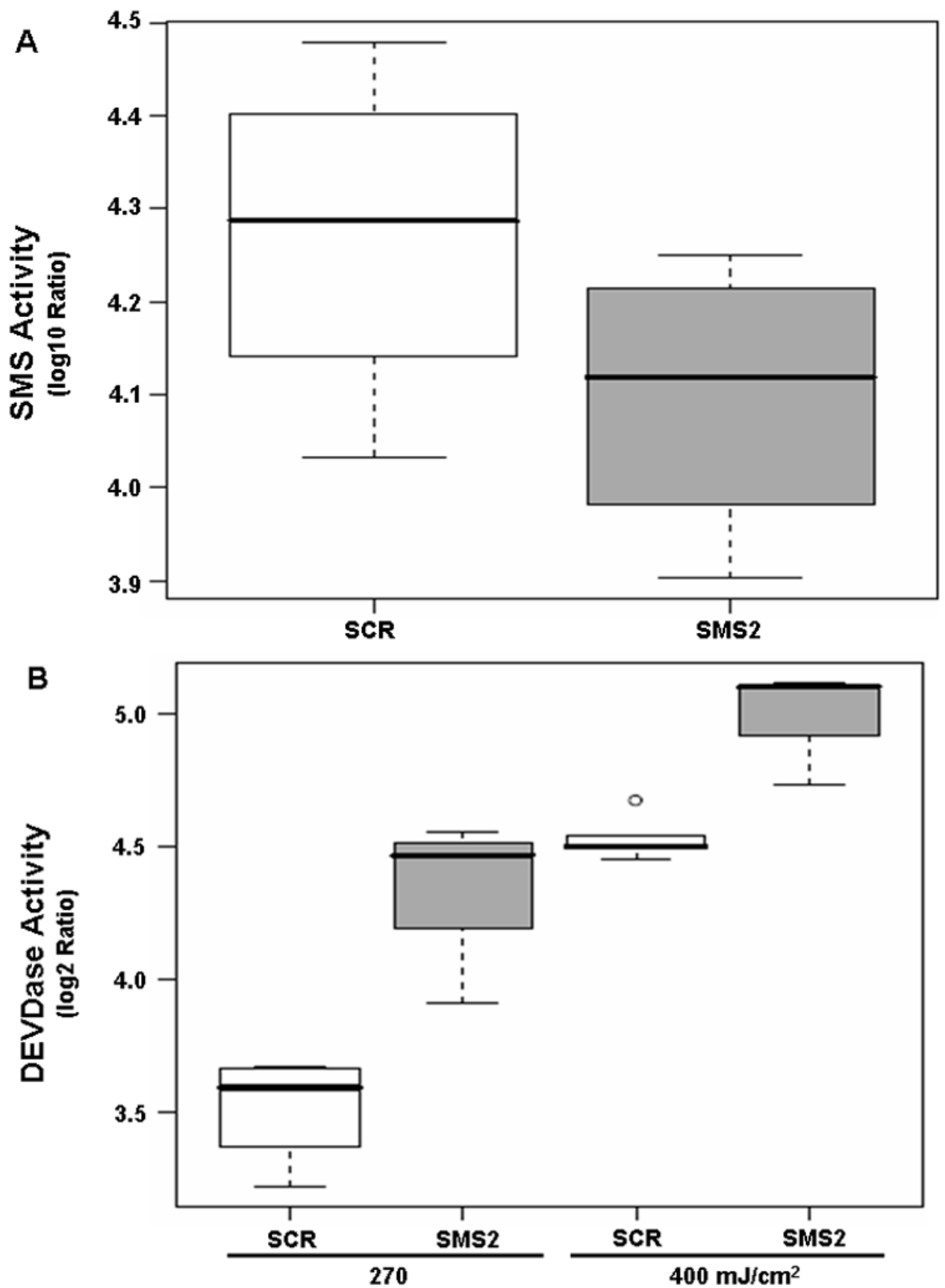

Figure 6.

(A) SMS is downregulated in SMS2 siRNA-transfectants. Cells were transfected with SMS2or scrambled siRNA as described in "Material and Methods". Two days after transfection, cells were collected and seeded in fresh growth medium with or without Pc 4 (200 nM). Following overnight exposure to Pc 4, cells were harvested, and the enzyme activity was determined in cell lysates as described in "Material and Methods". (B) PDT-triggered activation of DEVDase is promoted in SMS2 siRNA-transfected Jurkat cells. Forty-eight hours after transfection, the cells were treated overnight with Pc $4(200 \mathrm{nM})$, irradiated at the indicated light fluences and then incubated for $2 \mathrm{~h}$. After the cells were harvested, DEVDase activity was measured in cell lysates spectrofluorimetrically using DEVD-AMC as the substrate. Three-five independent 
determinations of the enzyme were performed. The Y-axis shows $\log 2$ ratios for the enzyme as a function of both group and dose variable. SCR, scrambled siRNA-transfectants; SMS2, SMS2 siRNA -transfectants. 
Table 1

Statistical analysis of sphingomyelin levels in growing Jurkat cells transfected with scrambled siRNA- or SMS1 siRNAtransfected cells and given Pc 4 only but no light.

\begin{tabular}{lcc}
\hline & FCG & pGroupFDR \\
\hline C20-SM & 0.59 & $\mathbf{0 . 0 1 5}$ \\
C16-SM & 0.65 & $\mathbf{0 . 0 1 6}$ \\
C22:1-SM & 0.60 & $\mathbf{0 . 0 1 6}$ \\
C18-SM & 0.66 & $\mathbf{0 . 0 1 6}$ \\
C14-SM & 0.72 & $\mathbf{0 . 0 1 6}$ \\
C24:1-SM & 0.72 & $\mathbf{0 . 0 2 9}$ \\
C22-SM & 0.63 & $\mathbf{0 . 0 4 6}$ \\
C24-SM & 0.63 & $\mathbf{0 . 0 4 6}$ \\
C26:1-SM & 0.54 & 0.109 \\
C26-SM & 0.48 & 0.109 \\
C18:1-SM & 0.88 & 0.321 \\
C20:1-SM & 0.93 & 0.645 \\
\hline
\end{tabular}

The FCG is the estimated fold-change between two cell types (groups) regarding the sphingomyelin (SM) response. The pGroupFDR is the adjusted pvalue using false discovery rate algorithm. Bold type indicates statistical significance. 
Table 2

Statistical analysis of responses of sphingolipids to PDT in scrambled siRNA- and SMS1 siRNA-transfected cells.

\begin{tabular}{|c|c|c|c|c|}
\hline & FCG & FCD & pGroupFDR & pDoseFDR \\
\hline C26Ceramide & 1.74 & 1.21 & $6.4 \mathrm{E}-08$ & 2.39E-06 \\
\hline C24Ceramide & 1.35 & 0.99 & $1.2 \mathrm{E}-05$ & $5.20 \mathrm{E}-01$ \\
\hline C24:1Ceramide & 1.36 & 1.05 & 2.7E-04 & $1.07 \mathrm{E}-01$ \\
\hline C22Ceramide & 1.56 & 1.10 & 2.7E-04 & 4.86E-02 \\
\hline C26:1Ceramide & 1.45 & 1.48 & $1.0 \mathrm{E}-03$ & 4.71E-09 \\
\hline C16Ceramide & 1.16 & 1.27 & $7.0 \mathrm{E}-02$ & 6.13E-08 \\
\hline C22:1Ceramide & 1.34 & 2.02 & $7.1 \mathrm{E}-02$ & $4.34 \mathrm{E}-10$ \\
\hline C20Ceramide & 1.33 & 1.78 & $7.1 \mathrm{E}-02$ & 8.76E-09 \\
\hline C18:1Ceramide & 1.20 & 1.47 & $8.2 \mathrm{E}-02$ & $1.00 \mathrm{E}-08$ \\
\hline C20:1Ceramide & 1.26 & 1.97 & $2.7 \mathrm{E}-01$ & 3.84E-07 \\
\hline C18Ceramide & 1.10 & 1.93 & $3.6 \mathrm{E}-01$ & 2.17E-12 \\
\hline C14Ceramide & 1.00 & 1.50 & $9.6 \mathrm{E}-01$ & 3.50E-11 \\
\hline DHC24:1-Cer & 1.67 & 1.77 & 2.2E-02 & 8.71E-08 \\
\hline DHC24-Cer & 1.40 & 1.23 & 2.2E-02 & 4.42E-04 \\
\hline DHC22-Cer & 1.27 & 1.32 & 2.2E-02 & $1.20 \mathrm{E}-07$ \\
\hline DHC22:1-Cer & 1.39 & 1.25 & $1.6 \mathrm{E}-01$ & 8.16E-03 \\
\hline DHC14-Cer & 1.23 & 2.36 & $2.6 \mathrm{E}-01$ & 2.18E-12 \\
\hline DHC16-Cer & 1.27 & 2.17 & $2.6 \mathrm{E}-01$ & 4.40E-09 \\
\hline DHC20:1-Cer & 1.25 & 1.78 & $2.6 \mathrm{E}-01$ & $1.08 \mathrm{E}-06$ \\
\hline DHC18:1-Cer & 0.81 & 1.70 & $2.6 \mathrm{E}-01$ & 1.12E-07 \\
\hline DHC18-Cer & 1.16 & 3.98 & $4.8 \mathrm{E}-01$ & $2.71 E-13$ \\
\hline DHC26-Cer & 1.08 & 1.13 & $5.4 \mathrm{E}-01$ & $1.98 \mathrm{E}-02$ \\
\hline DHC26:1-Cer & 1.05 & 1.45 & $8.1 \mathrm{E}-01$ & $1.11 E-05$ \\
\hline DHC20-Cer & 1.01 & 1.89 & $9.7 \mathrm{E}-01$ & 4.36E-05 \\
\hline C14-SM & 1.33 & 0.93 & 3.4E-02 & 4.07E-01 \\
\hline C20:1-SM & 0.83 & 1.00 & $6.3 \mathrm{E}-02$ & $9.57 \mathrm{E}-01$ \\
\hline C16-SM & 1.20 & 0.97 & $6.3 \mathrm{E}-02$ & $7.09 \mathrm{E}-01$ \\
\hline C18-SM & 1.16 & 0.95 & $1.1 \mathrm{E}-01$ & $5.31 \mathrm{E}-01$ \\
\hline C26:1-SM & 1.26 & 0.95 & $1.1 \mathrm{E}-01$ & $6.14 \mathrm{E}-01$ \\
\hline C18:1-SM & 1.24 & 1.02 & $1.2 \mathrm{E}-01$ & $8.87 \mathrm{E}-01$ \\
\hline C22-SM & 0.92 & 0.94 & $4.3 \mathrm{E}-01$ & 4.07E-01 \\
\hline $\mathrm{C} 20-\mathrm{SM}$ & 1.09 & 1.01 & $4.4 \mathrm{E}-01$ & $8.87 \mathrm{E}-01$ \\
\hline C24:1-SM & 0.94 & 0.98 & $4.4 \mathrm{E}-01$ & $7.70 \mathrm{E}-01$ \\
\hline C22:1-SM & 0.97 & 0.96 & $8.6 \mathrm{E}-01$ & $5.76 \mathrm{E}-01$ \\
\hline C24-SM & 0.99 & 0.95 & $9.7 \mathrm{E}-01$ & $4.07 \mathrm{E}-01$ \\
\hline C26-SM & 1.00 & 1.00 & 9.7E-01 & $9.57 \mathrm{E}-01$ \\
\hline Sph & 2.35 & 1.05 & $4.4 \mathrm{E}-04$ & $6.03 \mathrm{E}-01$ \\
\hline Sph-1P & 0.54 & 1.28 & 2.6E-02 & 4.72E-02 \\
\hline DHSph-1P & 0.75 & 1.64 & $3.6 \mathrm{E}-01$ & $2.63 \mathrm{E}-03$ \\
\hline DHSph & 1.22 & 1.64 & $3.6 \mathrm{E}-01$ & $1.35 \mathrm{E}-04$ \\
\hline
\end{tabular}

DHC24:1-Cer, C24:1-dihydroceramide; C14-SM, C14-sphingomyelin. Similar abbreviations were used for other dihydroceramides and sphingomyelins, respectively. Sph, sphingosine; Sph-1P, sphingosine-1-phosphate, DHSph-1P, dihydrosphingosine-1-phosphate; DHSph, dihydrosphingosine.

The FCG is the estimated fold-change between cell lines (groups) regarding a sphingolipid response, assuming a constant PDT dose. The FCD is the estimated fold-change in response to each 100 units of dose (light fluence), regardless of the group. The pGroupFDR and the pDoseFDR are corresponding adjusted p-values using false discovery rate algorithm. Bold type indicates statistical significance. 
Table 3

Statistical analysis of the response of DEVDase to PDT in SMS1 siRNA- and SMS2 siRNA-transfectants compared to scrambled (control) siRNA-transfectants.

\begin{tabular}{lccc}
\hline & FCG & FCD & pGroupFDR \\
\hline SMS1 siRNA & 1.65 & 1.94 & $\mathbf{1 . 4 8 E - 0 5}$ \\
SMS2 siRNA & 1.53 & 1.61 & $\mathbf{1 . 0 8 E - 0 4}$ \\
\hline
\end{tabular}

The FCG is the estimated fold-change between SMS1 siRNA-transfectants and their corresponding controls, or between SMS2 siRNA-transfectants and their corresponding controls regarding the DEVDase response, assuming a constant PDT dose. The FCD is the estimated fold-change in response to each 100 units of dose (light fluence), regardless of the group (cell type). The pGroupFDR and the pDoseFDR are corresponding adjusted p-values using false discovery rate algorithm. Bold type indicates statistical significance. 